\title{
The Propagation of Hydromagnetic Cylindrical Shock Waves in Weak Magnetic Field, With A Self-Gravitating Gas
}

\author{
Dr. Sarvesh Chandra Yadav
}

Assistant Professor and Head Department of Physics C.L. Jain P.G. College, Firozabad, India

$\begin{array}{ll} & \text { ABSTRACT } \\ & \text { The effects of overtaking disturbances behind the flow on the propagation of } \\ \text { Article Info } & \text { diverging cylindrical shock Waves through an ideal gas in presence of a } \\ \text { Volume 8, Issue 6 } & \text { magnetic field having } H_{Z_{0}}=\text { constant }=H_{\theta_{0}} \text { and an Initial density distribution } \\ \text { Page Number : 311-323 } & \rho_{0}=\rho^{\prime} r^{-\omega} \text { where } \omega \text { is a constant, } \rho^{\prime} \text { is the density at the plane / exes of } \\ \text { Publication Issue } & \text { symmetry: The analytical formula for flow variables representing both the } \\ \text { November-December-2021 } & \text { position form viz; weak and strong cases at shock waves have been obtained. } \\ & \text { Their numerical estimates at permissible shock front locations have been } \\ \text { Article History } & \text { obtained. } \\ \text { Accepted : 01 Dec 2021 } & \text { There numerical estimates at permissible shock front location's have been } \\ \text { Published : 08 Dec 2021 } & \text { Calculated and compared with earlier result describing in Free Propagation } \\ & \text { through figures. After inclusion of E.O.D. noted that there is no change at flow } \\ & \text { variable with parameters } r, \beta^{2}, \omega \text { and } \xi \text {. However, the trends of variation with } \\ & \text { propagation distance } r \text {, for shock strength, shock velocity and particle velocity } \\ & \text { are not change in case of weak shock with work Magnetic field(wswmf). } \\ & \text { Keywords : E.O.D } \rightarrow \text { Effect of overtaking disturbances, } \\ & \rho_{0}=\rho^{\prime} r^{-\omega} \rightarrow \text { Density Distributions } \\ & H_{Z_{0}} \rightarrow \text { Azimuthal component of magnetic field } \\ & H_{\theta_{0}} \rightarrow \text { Axial comp. of Magnetic field } \\ & \text { WSWMF } \rightarrow \text { Weak shock weak magnetic field. } \\ & \end{array}$

\section{INTRODUCTION}

Using CCW method. by including the e.o.d behind the flow of cylindrical weak shock wave in weak magnetic field in a self gravitating gas. CCW method for shock wave dynamics that it's application is useful unless the result obtained are compared with other numerical methods Witham has ruled out this limitation and claimed it's correctness Rosciszewski and oshima formulation of the error involved in using the CCW description by integrating two neighboring overtaking characteristics.

The characteristics method which works for weak and strong condition of shock. 
The C.O.D behind the flow on the propagation of diverging cylindrical shock wave through an Ideas and electrically perfectly conducting self gravitating gas in presence of a magnetic field having constant azimuthal $\left(H_{z_{0}}\right)$ and axial components and magnetic fields for shock weak. The density in the unperturbed state has been $\rho_{0}=\rho^{\prime} r^{-\omega}$.The case of weak shock explored under weak magnetic field

The expression for flow variables have been computed only at psfl with Parameter r, $\beta^{2}, \mathrm{D}, \beta_{2}^{2} \omega$ and $\xi$ also compared with result obtained through Free Propagation in Figures (1-5)

\subsection{Fundamental Equation :-}

The equation governing the cylindrical flow at the gas under the influence of its own gravitating and magnetic field having constant axial and azimuthal components of Magnetic field are written as

$\frac{\partial u}{\partial t}+u \frac{\partial u}{\partial r}+\frac{1}{\rho} \frac{\partial \rho}{\partial r}+\frac{\mu}{2 \rho} \frac{\partial}{\partial r}\left(H_{0}^{2}+H_{z}^{2}\right)+\frac{\mu}{\rho} \frac{H_{0}^{2}}{r}+\frac{G m}{r^{2}}-0$,

$\frac{\partial u}{\partial t}+u \frac{\partial \rho}{\partial r}+\rho \frac{\partial u}{\partial r} \rho \frac{u}{r}=0$,

$\frac{\partial \rho}{\partial t}+u \frac{\partial \rho}{\partial r}+\gamma p \frac{\partial u}{\partial r}+\gamma p \frac{u}{r}=0$

$\frac{\partial H_{\theta}}{\partial t}+u \frac{\partial H_{\theta}}{\partial r}+H_{\theta} \frac{\partial u}{\partial r}=0$,

$\frac{\partial H_{z}}{\partial t}+u \frac{\partial H_{z}}{\partial r}+H_{z} \frac{\partial u}{\partial r}+H_{z} \frac{u}{r}=0, \quad \frac{\partial m}{\partial r}-2 \pi \rho r=0$

Where $r \rightarrow$ radial co-ordinate $u, \rho, \rho H_{Z}, H_{\theta}, \mu$ and $\mathrm{m}$ are. respectively, the particle velocity, thy density. the pressure, azimuthal and axial components at magnetic field permeability at gas, mass inside a cylinder of unit cross-section and unit radius and unit length.

$a_{0}^{2}=\frac{\gamma p_{o}}{\rho_{o}}, \gamma \rightarrow$ Adiabatic index of the gas

\subsection{Boundary Condition :-}

The magnetohydrodynamic conditions can be written in terms of single parameter.

$$
\begin{gathered}
\xi=\frac{\rho}{\rho} \text { as } \rho=\rho_{0} \xi, \\
H=H_{0} \xi, \quad u=\frac{\xi-1}{\xi} U, \\
U^{2}=\frac{2 \xi}{(\gamma+1)-(\gamma-1) \xi}\left[a_{0}^{2}+\frac{b_{0}^{2}}{2}\{(2-\gamma) \xi+\gamma\}\right]
\end{gathered}
$$

where ' $\mathrm{o}$ ' stande for the state immediately ahead of the shock front, $\mathrm{U}$ is the shock velocity, $a_{0}$ is the sound speed $a_{0}=\sqrt{\frac{\gamma p_{0}}{\rho_{0}}}$ and alfven speed $\left(\frac{\mu H_{0}^{2}}{\rho_{0}}\right)^{\frac{1}{2}}$.

1.4 Weak Shock :-For every weak shock the parameter $\xi$ is written as

$\frac{\rho}{\rho_{0}}=1+\varepsilon=\xi$

Where $\varepsilon(r)$ is another parameter which is negligible in comparison with unity, i.e. $\varepsilon(r)<<1$, 
Now we consider to weak magnetic field $b_{0}^{2}<<a_{0}^{2}$, i.e., $\mu H_{0}^{2} / \gamma p_{0}<<1$,

by using the boundary condition (2) for every weak chock reduce to

$\rho=\rho_{0}(1+\varepsilon), \quad H_{\theta}=H_{\theta_{0}}(1+\varepsilon), H_{z}=H_{z_{0}}(1+\varepsilon)$

$U=\left(1+\frac{\gamma+1}{4} \varepsilon\right) a_{0}, \quad p=p_{0}(1+\gamma \varepsilon)$ and $u=\varepsilon a_{0}$

1.5 CHARACTERISTIC EQUATION: For diverging shock. the characteristic form of the system of equation (1) is easily obtained by forming a linear combination of (1) and (3) equation of the system of equation (1) in only one direction in $(r, t$,$) plane Equation (1) and (3) of the system can be written as$

$\rho \frac{\partial u}{\partial t}+\rho u \frac{\partial u}{\partial r}+\frac{\partial t}{\partial r}-\rho \frac{G m}{r^{2}}=0$,

$\frac{\partial p_{t}}{\partial t}+u \frac{\partial p_{t}}{\partial r}+\rho c^{2} \frac{\partial u}{\partial r}+\rho \frac{c^{2} u}{r}=0$

$p_{1}=p+\frac{u}{2}\left(H_{0}^{2}+H_{z}^{2}\right)+\int \mu H_{\theta}^{2} \frac{d r}{r}$

is total pressure including magnetic pressure and

$C^{2}=a^{2}+b^{2}=\frac{\gamma p}{\rho}+\frac{\mu}{\rho}\left(H_{\theta}^{2}+H_{z}^{2}\right)$

with the help of above equation we get characteristic equation on

$d p+\mu H_{\theta} d H_{\theta}+\mu H_{z} d H_{z}+\rho c d u+\mu H_{\theta}^{2} \frac{d r}{r}+\rho c^{2} \frac{u}{u+c} \frac{d r}{r}+\rho c \frac{G m}{u+c} \frac{d r}{r^{2}}=0$

In order to estimate the strength of overtaking disturbances an independents $C_{+}$characteristic is considered the differential relation valid across $C_{+}$disturbances is obtained by replaced c by $-^{-c}$ in equation (8) and written as

$d p+\mu H_{\theta} d H_{\theta}+\mu H_{z} d H_{z}+\rho c d u+\mu H_{\theta}^{2} \frac{d r}{r}+\rho c^{2} \frac{u}{u+c} \frac{d r}{r}+\rho c \frac{G m}{u+c} \frac{d r}{r^{2}}=0$

equation (9) represent characteristic form of equation (1) for Conversing shock.

\subsection{ANALYTICAL RELATIONS FOR FLOW VARIABLES :-}

Considered on initial density distribution of the form viz,

$$
\rho_{0}=\rho^{1} r^{-w}
$$

For the equilibrium state of the as is assumed

$$
\begin{aligned}
& \frac{\partial}{\partial t}=0=u \\
& H_{z_{0}}=H_{\theta_{0}},
\end{aligned}
$$

using (ii) and First equation of the system of equation (1), the hydrostatic equilibrium prevailing in front of shock can be written as 


$$
\begin{aligned}
& \frac{1}{p_{0}} \frac{d p_{0}}{d r}+\frac{\mu}{2 \rho_{0}} \frac{d}{d r}\left(H_{Z_{0}}^{2}+H_{\theta_{0}}^{2}\right)+\frac{1}{\rho_{0}} \frac{\mu H_{\theta_{0}}^{2}}{r}+\frac{G m}{r^{2}}=0 \\
& \frac{1}{\rho_{0}} \frac{d p_{0}}{d r}+\frac{1}{\rho_{0}} \frac{\mu H_{\theta_{0}}^{2}}{r}+\frac{G m}{r^{2}}=0
\end{aligned}
$$

From the sixth equation of (1) can be written as

$$
\begin{aligned}
& m=2 \pi \rho^{1} \int r^{1-w} \\
& m=\frac{2 \pi \rho^{1} r^{2-w}}{2-w}
\end{aligned}
$$

from (10), (12) and (13) we get

$$
\frac{p_{0}}{G \rho^{12}}=K-\beta_{2}^{2} D \log r-K_{1} r^{1-2 w}
$$

why $\mathrm{K}$ is constant

$$
\frac{a_{0}}{a^{1}}=\left(\frac{\gamma r^{w}}{D}\left(K-\beta_{2}^{2} D \log r-K_{1} r^{1-2 w}\right)\right)^{\frac{1}{2}}
$$

why $\quad K_{1}=\frac{2 \pi}{(1-2 w)(2-w)}$,

$D=\frac{a^{2}}{G \rho^{2}} \beta_{2}^{2}=\frac{\mu H_{\theta_{0}}^{2}}{\gamma p}$

$\rho^{\prime}$ it the density at the plane of symmetry in unperturbed state and G is universal Gravitational constant. Variations in the pressure in the unperturbed medium given by equation (14) with the Propagation distance $\mathbf{r}$.

1.7 WEAK SHOCK WITH WEAKMANETIC FIELD (WSWMF): Putting the shock condition (4) in to equation (8) and using the equation (12) and neglected the second and higher order term of $\varepsilon(r)$ since $\varepsilon(r)<<1$, we get $\frac{d \varepsilon}{\varepsilon}+\frac{1}{2}\left(1-\frac{1}{2} \frac{\mu H_{0}^{2}}{\gamma p_{0}}\right)\left(\frac{d p_{0}}{p_{0}}+\frac{d a_{0}}{a_{0}}+\frac{d r}{r}+\frac{2 \mu H_{\theta_{0}}^{2}}{\gamma p^{0}} \frac{d r}{r}\right)=0$

The validity condition of above relation is $\frac{\mu H_{0}^{2}}{\gamma \rho_{0}}<<1$ and this equation can be re-written as

$$
\frac{d \varepsilon}{\varepsilon}+\frac{1}{2}\left(1-\frac{\beta^{2} p^{1}}{2 p^{0}}\right)\left(\frac{d p_{0}}{p_{0}}+\frac{d a_{0}}{a_{0}}+\frac{d r}{r}+\frac{\beta_{2}^{2} p^{1}}{p_{0}} \frac{d r}{r}\right)=0
$$

Substituting the values of $\frac{d p_{0}}{p_{0}}$, and $\frac{d p_{0}}{p_{0}}$, into equation (17) and on integration we get

$$
\begin{aligned}
& \varepsilon(r)=K_{12} r^{K 11} \ell \cdot\left(K_{13}\right) \\
& \varepsilon(r)=K_{12} r^{K 11} \exp .\left(K_{13}\right)
\end{aligned}
$$

where $K_{12} \rightarrow$ integration, constant 


$$
\begin{aligned}
K_{13}=K_{6} r+K_{7} r^{1-2 w}+K_{8} \frac{r^{2-2 w}}{2-2 w}+K_{9} \frac{r^{2(1-2 w)}}{2(1-2 w)}+K_{10} \frac{r^{3-4 w}}{3-4 w} \\
K_{3}=-\frac{B_{2}^{2} D}{K^{2}}, K_{4}=-\left\{(1-2 w)+\frac{B_{2}^{2} D}{K}+(1-2 w) \frac{B_{2}^{2} D}{K} \log r\right\} \\
K_{5}=-(1-2 w)\left(\frac{K_{1}}{K}\right)^{2}, \\
K_{6}=-\frac{3}{4} K_{3}+\frac{3 B^{2} D}{8 \gamma K} K_{3,} \\
K_{7}=-\frac{3}{4} K_{4}+\frac{3 \beta^{2} D}{8 \gamma K} K_{4}+\frac{3 \beta^{2} D \beta_{2}^{2}}{8 \gamma K^{2}} K_{4} \log r+\left(1+\frac{w}{2}\right) \frac{\beta^{2} D K_{1}}{4 \gamma K^{2}}+\frac{2 \beta_{2}^{2} D K_{1}}{\gamma K^{2}} \\
K_{8}=-\frac{3}{8} \frac{\beta^{2} D K_{1}}{\gamma K^{2}}-\frac{3}{4} K_{4}+\frac{3}{8} \frac{\beta^{2} D}{\gamma K^{2}} K_{5}+\frac{3}{4} \frac{\beta^{2} D \beta_{2}^{2}}{4 \gamma K^{2}} \log r K_{5} \\
K_{9}=\frac{3 \beta^{2} D K_{1} K_{4}}{8 \gamma K^{2}} \\
K_{10}=\frac{3}{8} \frac{\beta^{2} D K_{1} K_{5}}{8 \gamma K^{2}} \\
K_{11}=\left\{\left(1+\frac{w}{2}\right)\left(1+\frac{\beta^{2} D}{4 \gamma K}+\frac{\beta^{2} D^{2} \beta_{2}^{2}}{2 \gamma K^{2}} \log r\right)\right\}
\end{aligned}
$$

Remember that equation (17) in to (4) describes Free propagation using the equation (17) into (4) we get $d u_{-}=\varepsilon d a_{0}+a_{0} \varepsilon\left\{-\frac{1}{2}\left(1-\frac{\mu H_{0}^{2}}{\gamma p_{0}}\right)\left(\frac{d p_{0}}{p_{0}}+\frac{d a_{0}}{a_{0}}+\frac{d r}{r}+\frac{2 \mu H_{\theta_{0}}^{2}}{\gamma p_{0}} \frac{d r}{r}\right)\right\}$

On substituting the shock conditions (4) into (9) and using (12) we get

$\frac{d \varepsilon}{\varepsilon}+\frac{\gamma p_{0}}{\mu H_{0}^{2}}\left\{\frac{d p_{0}}{p_{0}}\left(\frac{1}{2}-\frac{2}{\gamma}\right)-\frac{d a_{0}}{a_{0}}-\frac{d r}{r}\right\}=0$

using the equation (20) and (4) we get

$$
d u_{+}=\varepsilon d a_{0}+a_{0}\left[\left(\frac{\gamma p_{0}}{\mu H_{0}^{2}}\right)\left\{\frac{d p_{0}}{p_{0}}\left(\frac{1}{2}-\frac{2}{\gamma}\right)-\frac{d a_{0}}{a_{0}} \frac{d r}{r}\right\} \varepsilon\right]
$$

Now in presence of both and disturbances the fluid velocity behind the shock will be related as

$$
d u_{-}+d u_{+}=\varepsilon d a_{0}+a_{0} \varepsilon
$$

from equation (19) (21) and (22) we get

$$
\frac{d \varepsilon}{\varepsilon}=\frac{d p_{0}}{p_{0}}\left[-\frac{1}{4}+\frac{3}{8} \frac{\mu H_{0}^{2}}{\gamma p_{0}}-\frac{\gamma p_{0}}{\mu H_{0}^{2}}\left(\frac{\gamma-4}{2 \gamma}\right)\right]+\frac{d r}{r}\left[-\frac{w}{4}-\frac{3}{2}+\frac{\mu H_{0}^{2}}{4 \gamma p_{0}}\left(1+\frac{w}{2}\right)-\frac{2 \mu H_{\theta 0}^{2}}{\gamma p_{0}}\right]
$$

Substituting the values of $\frac{d p_{0}}{p_{0}}, \frac{p_{0}}{p_{1}}$ and $\frac{d a_{0}}{a_{0}}$ into equation (23) and on integration

$$
\frac{\varepsilon(r)=K_{20} r^{K_{15}} \exp .\left(K_{21}\right)}{K_{20} \rightarrow \text { Integration constant }}
$$




$$
\begin{aligned}
& K_{21}=K_{14}+K_{16} \frac{r^{1-2 w}}{1-2 w}+K_{17} \frac{r^{2-2 w}}{2-2 w}+K_{18} \frac{r^{2-4 w}}{2-4 w}+K_{19} \frac{r^{3-4 w}}{3-4 w} \\
& K_{14}=-\frac{K_{3}}{4}+\frac{3 \beta^{2} D K_{3}}{8 \gamma K}+\frac{3}{8} \frac{D^{2} \beta_{2}^{2} K_{3}}{\gamma K_{2}} \beta^{2} \log r-K_{3} \frac{\gamma K}{D \beta^{2}}\left(\frac{\gamma-4}{2 \gamma}\right)+\frac{\gamma-4}{2 \gamma} \frac{K \gamma \beta_{2}^{2}}{\beta^{2}} \log r \\
& K_{15}=-\frac{w}{4}+\frac{3}{2}+\frac{\beta^{2} D}{4 \gamma K}+\left(1+\frac{w}{2}\right) \\
& K_{16}=-\frac{K_{4}}{4}+K_{4} \frac{3 \beta^{2} D}{8 \gamma K}+\frac{3 \beta^{2} D}{8 \gamma K}\left(D \beta_{2}^{2} \log r\right)-\left(\frac{\gamma-4}{2}\right) \frac{K}{\beta^{2} D}\left(1-\frac{D \beta_{2}^{2}}{K} \log r\right) \\
& K_{17}=\left\{\frac{3}{8} \frac{\beta^{2} D}{\gamma K}+\left(\frac{\gamma-4}{2}\right) \frac{1}{\beta^{2} D}\right\} K_{1} K_{3}-\frac{\gamma-4}{2} \frac{K}{\beta^{2} D}\left(1-\frac{\beta_{2}^{2} D}{K} \log r\right) \\
& K_{18}=K_{4}\left(-\frac{1}{4}+\frac{3 \beta^{2} D}{8 \gamma K} K_{1}\right) \\
& K_{19}=K_{5}\left(\frac{3 \beta^{2} D}{8 \gamma K^{2}}+\frac{\gamma-4}{2 \beta^{2} D} K_{1}\right)
\end{aligned}
$$

Equation (24) is the propagation parameter which includes the e.o.d behind the flow on the motion of shock.

\subsection{ANALYTICAL EXPRESSIONS FOR FLOW VARIABLES FOR WSWMF :-}

Substitution the equation (18) and (24) in to shock condition (4), we get respectively for FP and flow variable $h$ and included the e.o.d.

\section{Free propagation}

$$
\begin{aligned}
& \frac{U}{a_{0}}=\left[1+\frac{1+\gamma}{4}\left\{K_{12} r^{K 11} \exp \cdot\left(K_{13}\right)\right\}\right] \\
& \frac{U}{\sqrt{G \rho^{1}}}=\left[\left\{\gamma r^{w}\left(K-\beta_{2}^{2} D \log r-K_{1} r^{1-2 w}\right)\right\}^{\frac{1}{2}}\left\{1+\frac{1+\gamma}{4}\left(K_{12} r^{K 11} \exp \cdot K_{13}\right)\right\}\right] \\
& \frac{\rho}{G \rho^{\prime 2}}=\left[\left(K-\beta_{2}^{2} D \log r-K_{1} r^{1-2 w}\right)+\left\{1+\gamma\left(K_{12} r^{K 11} \exp \left(K_{13}\right)\right)\right\}\right] \\
& \frac{u}{\sqrt{G \rho^{1}}}=\left[\left\{\gamma r^{w}\left(K-\beta_{2}^{2} D \log r-K_{1} r^{1-2 w}\right)\right\}^{\frac{1}{2}}\left\{K_{12} r^{K 11} \exp \left(K_{13}\right)\right\}\right] \\
& \frac{\rho}{\rho}=r^{-w}\left[\left\{1+K_{12} r^{K 11} \exp \left(K_{13}\right)\right\}\right]
\end{aligned}
$$

EOD:-

$$
\begin{aligned}
& \frac{U}{a_{0}}=\left[1+\frac{1+\gamma}{4}\left\{K_{20} r^{K 15} \exp \cdot\left(K_{21}\right)\right\}\right] \\
& \frac{U}{\sqrt{G \rho^{1}}}=\left[\left\{\gamma r^{w}\left(K-\beta_{2}^{2} D \log r-K_{1} r^{1-2 w}\right)\right\}^{\frac{1}{2}}\left\{1+\frac{1+\gamma}{4}\left(K_{20} r^{K 12} \exp \cdot K_{21}\right)\right\}\right] \\
& \frac{\rho}{G \rho^{12}}=\left[\left(K-\beta_{2}^{2} D \log r-K_{1} r^{1-2 w}\right)+\left\{1+\gamma\left(K_{20} r^{K 15} \exp \left(K_{21}\right)\right\}\right]\right.
\end{aligned}
$$




$$
\begin{aligned}
& \frac{u}{\sqrt{G \rho^{1}}}=\left[\left\{\gamma r^{w}\left(K-\beta_{2}^{2} D \log r-K_{1} r^{1-2 w}\right)\right\}^{\frac{1}{2}}\left\{K_{20} r^{K 15} \exp \left(K_{21}\right)\right\}\right] \\
& \frac{\rho}{\rho^{1}}=r^{-w}\left[\left\{1+K_{20} r^{K 15} \exp .\left(K_{21}\right)\right\}\right]
\end{aligned}
$$

\section{Result and Discussion}

The present analysis gives a description of shock wave propagation with better degree of accuracy by including the e.o.d. behind the flow in CCW Method.

WSWMF. the flow variable are dependent on $r, \beta_{2}^{2}, D$ and $w$

Taking $\varepsilon(r)=0.24$ at $r=0.5$ for $=1.4$,

$\beta_{2}^{2}=0.10,0.15,0.20$ and $\beta^{2}=0.25,0.30 D=0.10,0+80$

$\omega=0.75,1.0,1.25$

The Numerical estimates of flow variables for both F.P and have including e.o.d.

given by expression 25-33 have been calculated only at psfl, the flow variables together with correction percentage due to e.o.d. variation in the flow variable with propagation distance $r$, have been shown in Figures (1-5)

"The shock strength increase where as the shock velocity, The pressure and density decrease with propagation distance r. Increase in $\beta_{2}^{2}$ and $\mathrm{D}$ leads to increase the shock strength and density whereas the shock velocity. The correction percentage lie between $-0.0479 \%$ to $1.4122 \%$

\subsection{GRAPH AND TABLE FOR FLOW VARIABLE}

Table 1 :- Flow variable for cylindrical WSWMF. in S.G.G. with Magnetic Field

$$
H_{Z_{0}}=\text { const }=H_{\theta_{0}}, \rho_{0}=\rho^{1} r^{-w}
$$

\begin{tabular}{|c|c|c|c|}
\hline S.No. & Variation of flow Variable & FD & E.O.D. \\
\hline 1 & shock strength $\left(\mathrm{u} / \mathrm{a}_{\mathrm{o}}\right)$ versus & Increase & Increase \\
& $r, \beta_{2}^{2}, \beta^{2} . w D$ & Decrease & Increase \\
\hline $\mathbf{2}$ & shock velocity versus & Decrease & Decrease \\
& $\left(\frac{U}{\sqrt{G \rho^{1}}}\right) r, \beta_{2}^{2}, D, w \beta^{2}$ & Increase & Increase \\
\hline 3 & $\left(\frac{\rho}{\sqrt{G \rho^{12}}}\right) r, \beta_{2}^{2}, D, \beta^{2}, w$. & Decrease & Decrease \\
& Pressure versus & Increase \\
\hline
\end{tabular}


Dr. Sarvesh Chandra Yadav Int J Sci Res Sci \& Technol. November-December-2021, 8 (6) : 298-310

$\begin{array}{lllllll}\text { CYLINDRICAL FLOW: WSWMF; }\left(\rho_{0}=\rho^{\prime} \mathrm{r}^{\mathrm{w}} \text { ") }\right. & \beta^{2} & \beta_{2}{ }^{2} & \mathrm{D} & \text { W } & \text { FP } & \text { EOD } \\ & 0.25 & 0.10 & 0.10 & 0.75 & \mathrm{a} & \mathrm{a}^{*} \\ \left(\mathrm{H}_{\mathbf{z}_{0}}=\text { Constant }=\mathrm{H}_{\theta_{0}}\right) & 0.25 & 0.10 & 0.10 & 0.75 & \mathrm{~b} & \mathrm{~b}^{*} \\ & 0.25 & 0.10 & 0.10 & 0.75 & \mathrm{c} & \mathrm{c}^{*} \\ & 0.25 & 0.10 & 0.10 & 1.00 & \mathrm{~d} & \mathrm{~d}^{*} \\ & 0.25 & 0.10 & 0.10 & 1.25 & \mathrm{e} & \mathrm{e}^{*} \\ & 0.25 & 0.10 & 0.8 & 0.75 & \mathrm{f} & \mathrm{f}^{*} \\ & 0.30 & 0.10 & 0.10 & 0.75 & \mathrm{~g} & \mathrm{~g}^{*} \\ & 0.35 & 0.10 & 0.10 & 0.75 & \mathrm{~h} & \mathrm{~h}^{*}\end{array}$

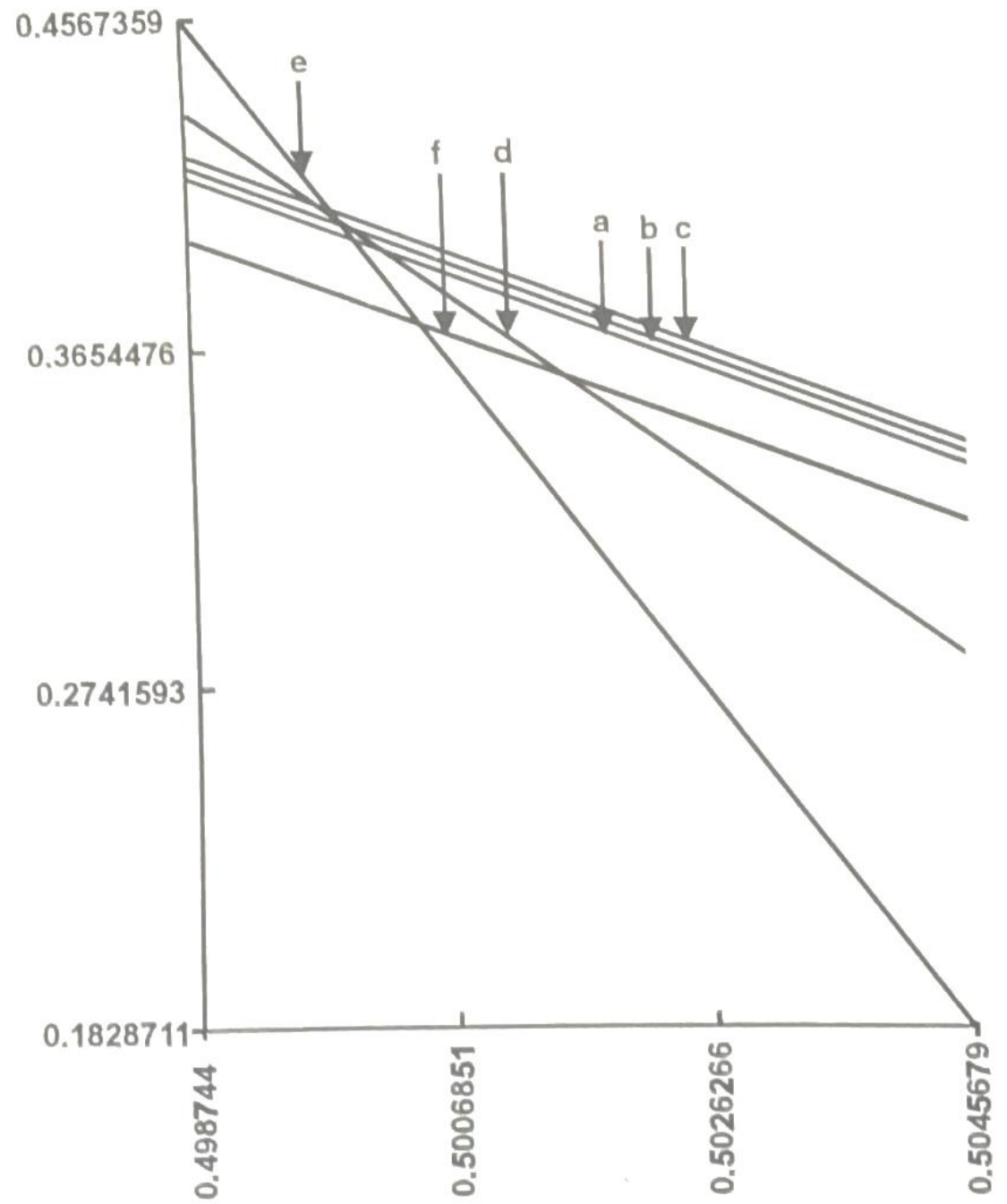
PROPAGATION DISTANCE (r) FOR CYLINDRICAL WSWMF IN A SGG. 


$$
\begin{array}{llllllll}
\text { CYLINDRICAL FLOW: WSWMF ; }\left(\rho_{0}=\rho^{\prime} \mathrm{r}^{-{ }^{*}}\right) & \beta^{2} & \beta_{2}{ }^{2} & \mathrm{D} & \text { W } & \text { FP } & \text { EO } \\
\left(\mathrm{H}_{\mathrm{Z}_{0}}=\text { Constant }=H_{0_{0}}\right) & 0.25 & 0.10 & 0.10 & 0.75 & \mathrm{a} & \mathrm{a}^{*} \\
& 0.25 & 0.15 & 0.10 & 0.75 & \mathrm{~b} & \mathrm{~b}^{*} \\
& 0.25 & 0.20 & 0.10 & 0.75 & \mathrm{c} & \mathrm{c}^{*} \\
& 0.25 & 0.10 & 0.80 & 0.75 & \mathrm{~d} & \mathrm{~d}^{*} \\
& 0.30 & 0.10 & 0.10 & 0.75 & \mathrm{e} & \mathrm{e}^{*} \\
& 0.35 & 0.10 & 0.10 & 0.75 & \mathrm{f} & \mathrm{f}^{*}
\end{array}
$$

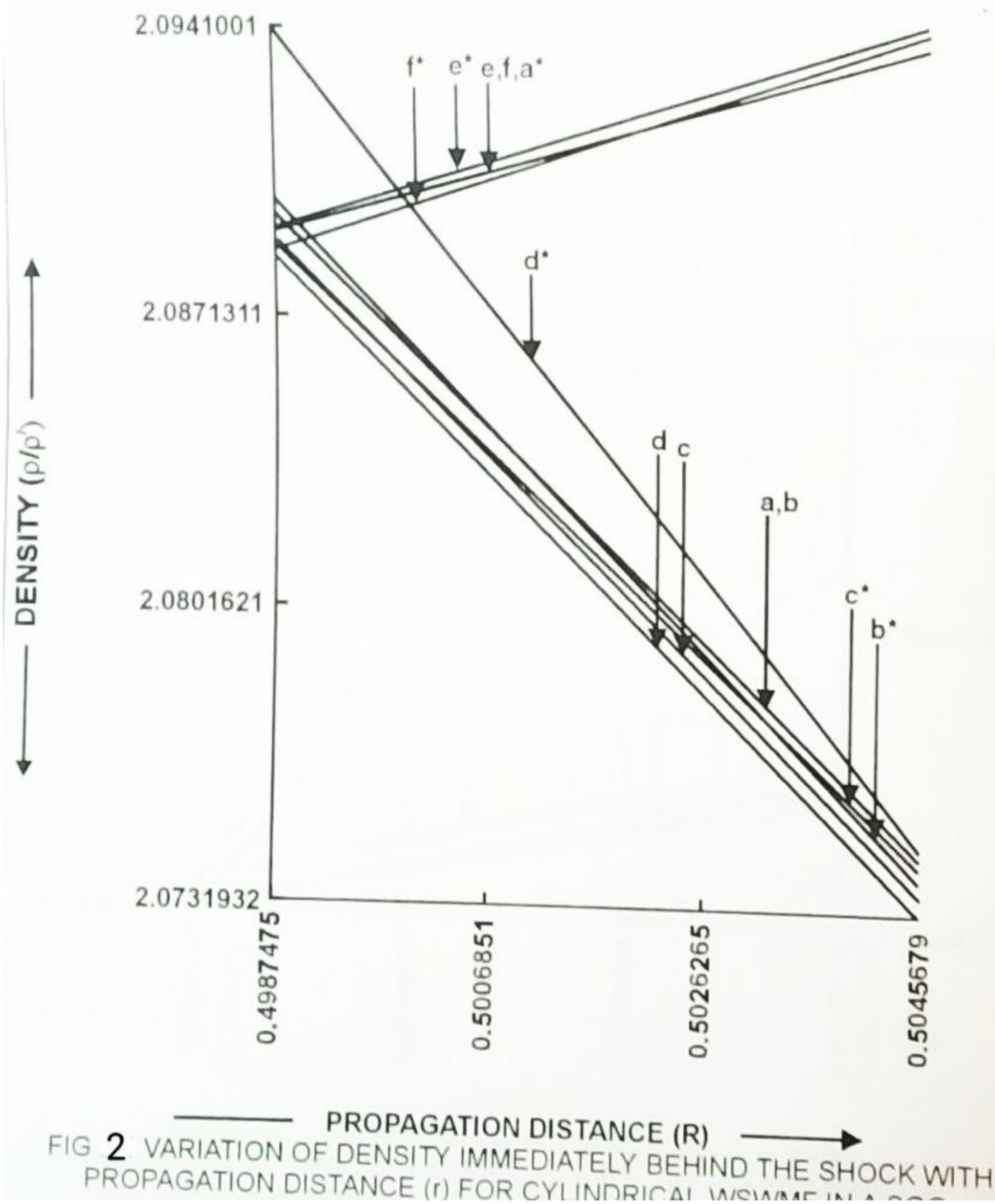


Dr. Sarvesh Chandra Yadav Int J Sci Res Sci \& Technol. November-December-2021, 8 (6) : 298-310

$$
\begin{array}{lllllll}
\text { CYLINDRICAL FLOW: WSWMF; }\left(\rho_{0}=\rho^{1} \mathrm{r}^{-w}\right) & \beta^{2} & \beta_{2}{ }^{2} & \mathrm{D} & \text { W } & \text { FP EOD } \\
\left(\mathrm{H}_{\mathrm{z}_{0}}=\text { Constant }=\mathrm{H}_{0_{0}}\right) & 0.25 & 0.10 & 0.10 & 1.0 & \mathrm{~g} & \mathrm{~g}^{*} \\
& 0.25 & 0.10 & 0.10 & 1.25 & \mathrm{~h} & \mathrm{~h}^{*}
\end{array}
$$

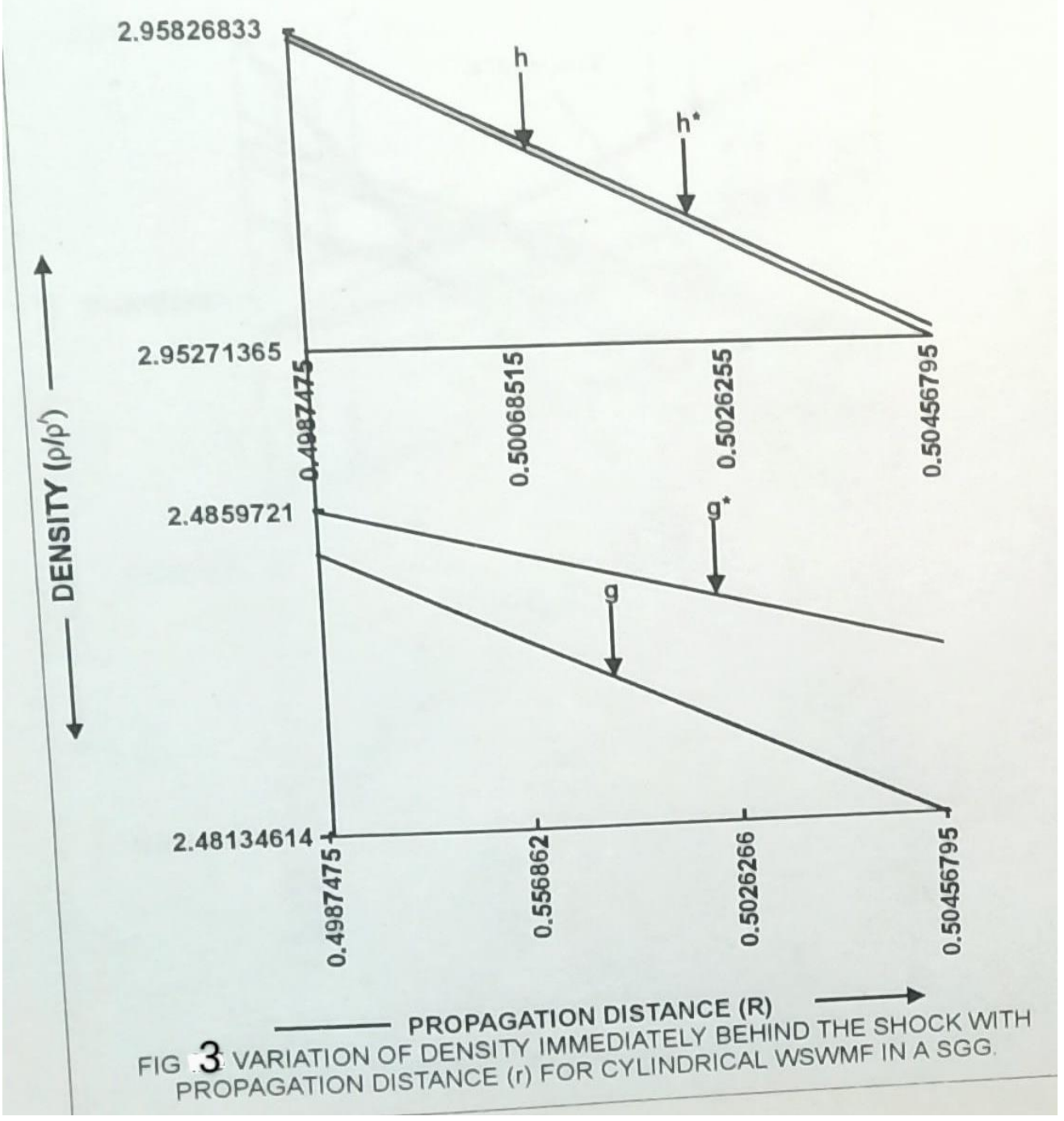


Dr. Sarvesh Chandra Yadav Int J Sci Res Sci \& Technol. November-December-2021, 8 (6) : 298-310
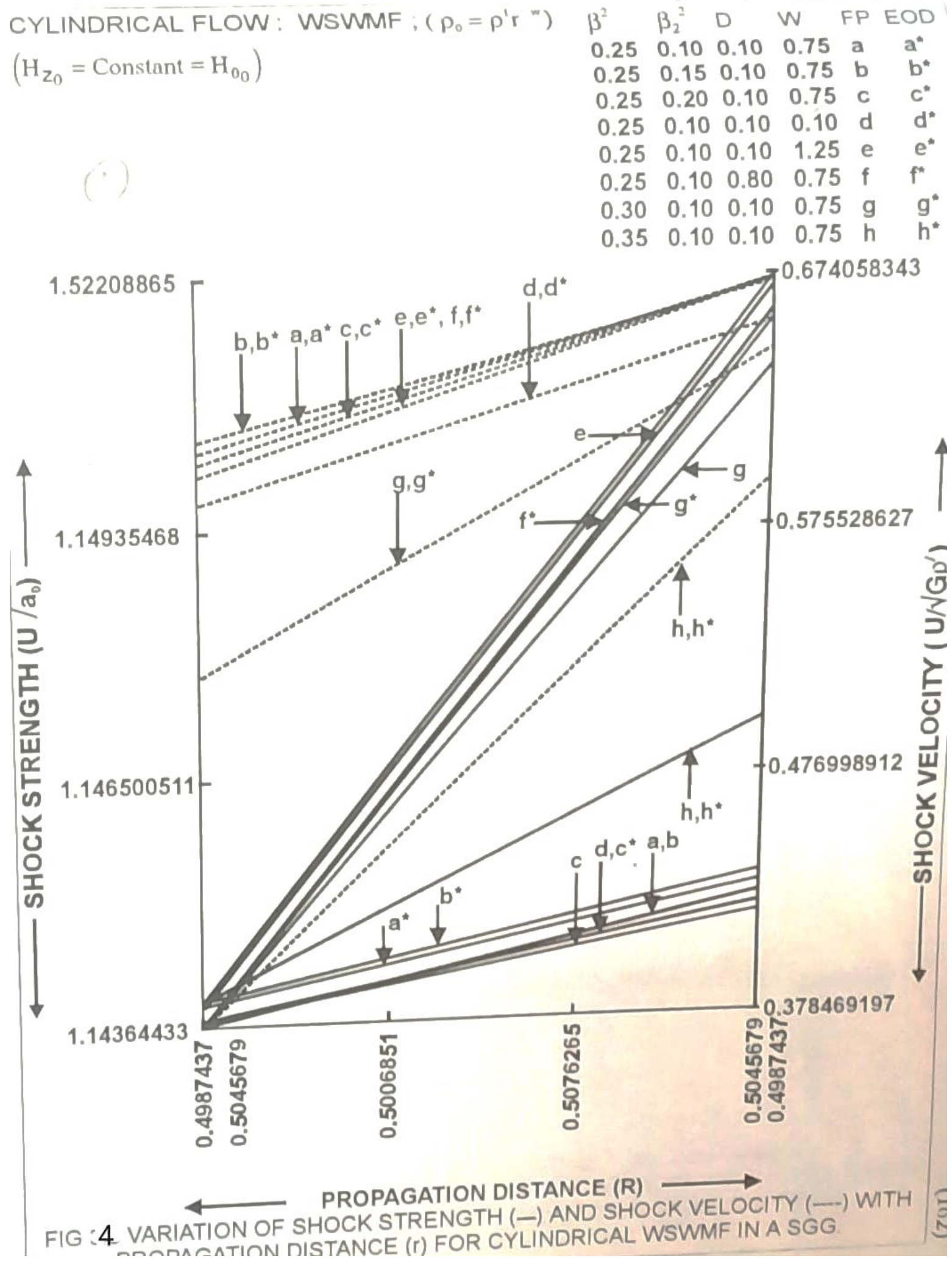


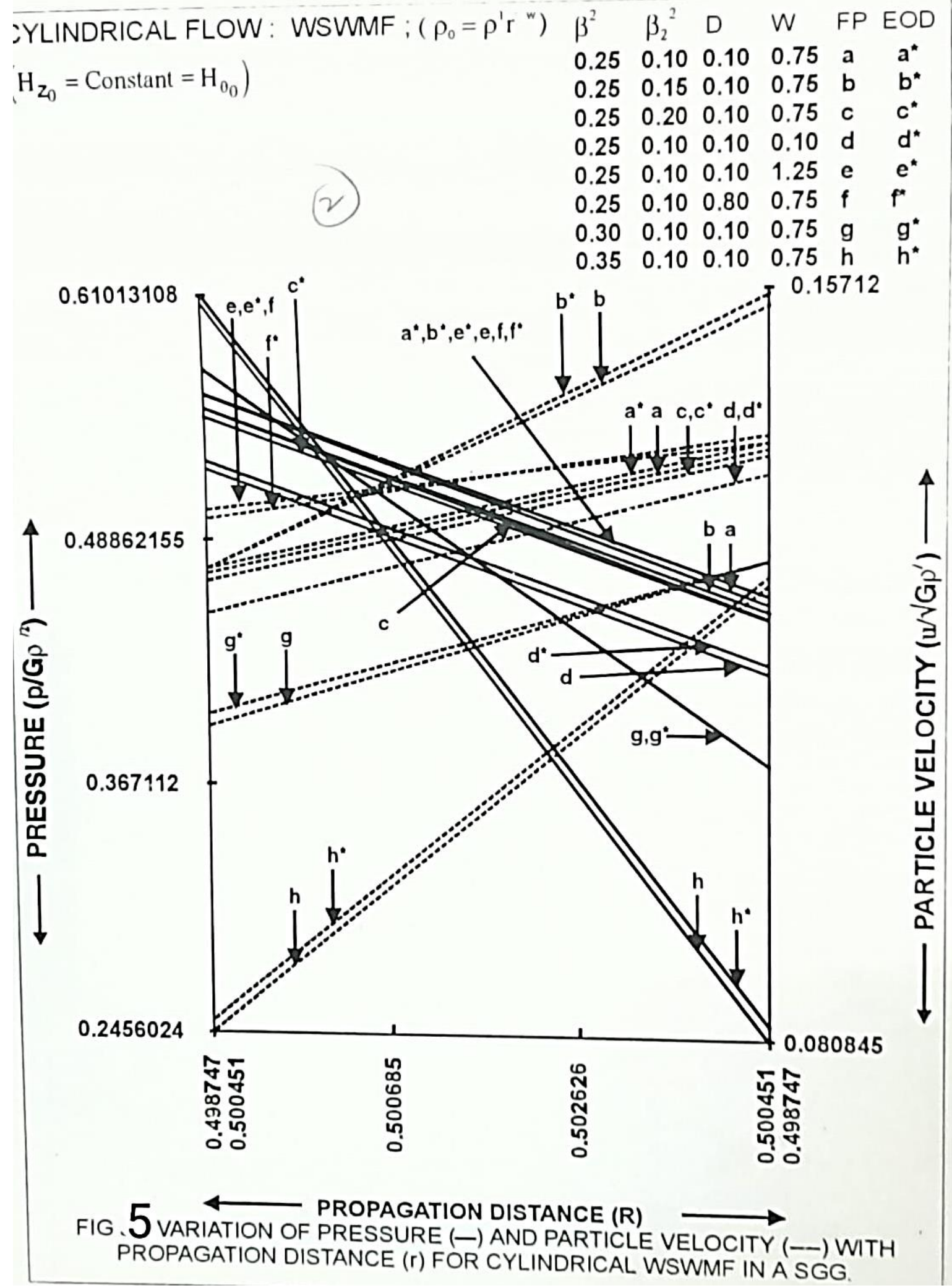

\section{REFERENCES}

[1]. Chester, W, "The quasi-cylindrical shock tube," Phil Mag., 45(7), 1293(1954).
[2]. Chisnell, R.F., "The normal motion of a shock wave through a non-uniform one-dimmensional medium," Proc. Roy. Soc. London, A 232. 350-370 (1955). 
[3]. Whitham, G.B., "The behavior of supersonic flow past a body of revolution ${ }^{\wedge \prime}$ Proc. Roy. Soc. (London), 203A, 571-581(1950).

[4]. Whitham, G.B., "On the propagation of shock waves through regions of non-uniform area or flow," J. Fluid Mech., 4, 337 (1958).

[5]. Hayes, W.D., "The propagation upward of the shock wave from a strong explosinon in the atmosphere," J. Fluid Mech.. 32, 317 (1968).

[6]. Kumar, S. and Chaturani, P., "Strong cylindrical shock in arotating gas," II Nuovo Cimenlo vol. 56B, 197-200 (1980).

[7]. Kumar, S., Kulshrestha, A.K, and Chaturani, P. "Plane andcylindrical shock in magnetogas dynamics," II NuovoCimento vol. 70B, 30-44 (1982).

[8]. Kumar, S. and Prakash. R. "Strong hydromagnctic cylindricalshock in a rotating gas," II. Nuovo Cimento B, Vol. 77, 191 -202(1983).

[9]. Kitgawa, S. and Kobayashi, M., "Examined their recordeddata of disturbance in the atmosphere" Proc. Int. Conf.Atmosphere and Space Elec. Montreux (1963).

[10]. Nath, G.,"Propagation of a strong cylindrical shock wave in arotational axisymmetric dusty gas with exponentially varyingdensity," Research in Astron. Astrophys. Vol. 10 No. 5, 445-460 (2010).

[11].Nath, G.," Propagation of a cylindrical shock wave in arotational axisymmetric isothermal flow of a non-ideal gas inmagnetogasdynamics," Ain Shams Engineering Journal Vol,3,393-01 (2012).

\section{Cite this article as :}

Dr. Sarvesh Chandra Yadav, "The Propagation of Hydromagnetic Cylindrical Shock Waves in Weak Magnetic Field, With A Self-Gravitating Gas ", International Journal of Scientific Research in Science and Technology (IJSRST), Online ISSN : 2395-602X, Print ISSN : 2395-6011, Volume 8 Issue 6, pp. 311-323, November-December 2021. Available at doi : : https://doi.org/10.32628/IJSRST218646 Journal URL : https://ijsrst.com/IJSRST218646 